\title{
Avaliação Térmica e Reológica do Ciclo de Cura do Pré-Impregnado de Carbono/Epóxi
}

\author{
Michelle L. Costa, Jane M. F. de Paiva, Edson C. Botelho, Mirabel C. Rezende \\ Divisão de Materiais, Instituto de Aeronáutica e Espaço, CTA
}

Resumo: Parâmetros cinéticos e reológicos relacionados com o ciclo de cura de um sistema de pré-impregnado de resina epóxi (F584)/fibra de carbono (prepreg) foram determinados pelo uso de análises térmicas (DSC e DMA) e reológicas. Os parâmetros determinados foram utilizados para avaliar o ciclo de cura hoje utilizado industrialmente no processamento de compósitos poliméricos pelo uso do prepreg em estudo. Dos resultados das análises térmicas foi constatado para o sistema estudado que a cinética de cura é de ordem $n=1,3$ e que a razão de aquecimento mais apropriada na obtenção de compósito polimérico é de $2,5^{\circ} \mathrm{C} / \mathrm{min}$, pois esta razão de aquecimento proporciona um ciclo de cura a temperaturas mais baixas, quando comparada às demais razões estudadas, 5 e $10^{\circ} \mathrm{C} / \mathrm{min}$, favorecendo uma cura mais homogênea e mais bem controlada.

Palavras-chave: Pré-impregnado, compósito polimérico, resina epóxi, cinética de cura, ciclo de cura, DSC, DMA, análises reológicas.

\section{Thermal and Rheological Evaluation of a Carbon/Epoxy Prepreg Cure Cycle}

Abstract: The kinetic and rheological parameters related to the cure cycle of an epoxy resin (F584)/carbon fiber preimpregnated (prepreg) system were determined by thermal and rheological analyses. The results were used to evaluate the cure cycle used nowadays in the composite processing industry, with the same prepreg system. Based on thermal analyses results it was verified that the curing of the epoxy resin follows a polymerization kinetic of $\mathrm{n}=1.3$ order and that the most adequate heating rate for the composite processing is equal to $2.5^{\circ} \mathrm{C} / \mathrm{min}$. This heating rate favors a cure cycle at lower temperatures, when compared to the other heating rates studied, 5 and $10{ }^{\circ} \mathrm{C} / \mathrm{min}$, allowing a more homogeneous, better controlled cure reactions.

Keywords: Prepreg, polymeric composite, epoxy resin, cure kinetic, cure cycle, DSC, DMA, rheological analyses.

\section{Introdução}

Uma das maneiras mais utilizadas pela indústria aeronáutica para produzir compósitos poliméricos é pelo uso de pré-impregnados (prepregs). Genericamente, o pré-impregnado é um produto intermediário, pronto para moldagem, e pode ser definido como sendo uma mistura (ou composição) de fibras de reforço com um determinado polímero termorrígido formulado ou termoplástico, em uma fração específica em massa ${ }^{[1,2]}$.

As matrizes poliméricas termorrígidas mais utilizadas na impregnação de fibras são as resinas epóxi, fenólica e poliimida. Também matrizes termoplásticas têm sido consideradas na obtenção de compósitos com fibras de vidro, carbono ou aramida, tanto na forma de tecidos quanto na de fitas unidirecionais (tapes $)^{[3]}$. A utilização de pré-impregnados no processamento de compósitos permite manter a integridade física do reforço durante o seu manuseio para a moldagem, controlar previamente a fração volumétrica de fibras e ajustar o grau de cura da matriz impregnante de modo a facilitar o processamento ${ }^{[2]}$.
A tecnologia de fabricação de pré-impregnados é controlada por poucas empresas fornecedoras da indústria aeronáutica, devido às formulações dos sistemas de resina visarem atender necessidades específicas em termos de propriedades mecânicas do compósito final (produto acabado). $\mathrm{O}$ acesso às informações sobre os sistemas de resina (formulações, aditivos, condições de preparação) e, conseqüentemente, as variações nas condições de processo, que possibilitem uma otimização do programa de tratamento térmico do pré-impregnado são limitadas, sendo abordadas com relação a sistemas de resina muito específicos ${ }^{[4-17]}$.

Excelentes trabalhos de revisão envolvendo as ordens de reação e a cinética de cura de resinas termorrígidas, principalmente a epóxi, têm sido encontrados na literatura, na tentativa de esclarecer as possíveis reações químicas que ocorrem durante a cura e que determinam a morfologia da resina que, por sua vez, definem as propriedades do polímero termorrígido curado $^{[4-22]}$. Entretanto, apesar da extensa pesquisa nessa área, numerosas questões envolvendo a relação processamento-morfologia-propriedades dos sistemas de resina mais utilizados na indústria aero- 
náutica ainda não foram respondidas. Somado a isto, ainda existe muito conflito entre os pesquisadores sobre os possíveis mecanismos de reação envolvidos na cura das resinas termorrígidas ${ }^{[-22]}$.

Uma variedade de técnicas experimentais tem sido utilizada para acompanhar as reações de cura de sistemas termorrígidos, sendo as mais estudadas as de termoanálises e as reológicas. A partir da calorimetria exploratória diferencial (DSC), da análise térmica dinâmico-mecânica (DMA) e da reologia é possível estabelecer a cinética de cura, tempos de gelificação $\left(t_{g e l}\right)$ e vitrificação, assim como, a viscosidade e a temperatura de transição vítrea $\left(T_{g}\right)$ de matrizes poliméricas $^{[4-8,23-37]}$.

Com o auxílio de técnicas dinâmico-mecânicas, tais como DMA e reologia de pratos paralelos, pode-se estudar os efeitos de tempo e temperatura sobre um material polimérico, os quais são muito importantes e devem ser considerados tanto na seleção da matéria-prima a ser utilizada, quanto na otimização do processamento de materiais com aplicações estruturais ou de engenharia. A capacidade de se "prever" os efeitos de tempo e temperatura sobre as propriedades mecânicas tem se tornado cada vez mais importante na área de polímeros e compósitos poliméricos, principalmente devido ao grande crescimento na substituição dos metais por esses materiais ${ }^{[38]}$. Dessa forma, as vantagens da utilização destas técnicas de monitoramento residem no conhecimento do comportamento polimérico desses materiais, apoiando o controle do processamento a ser utilizado, que por sua vez, influencia nas propriedades mecânicas e nas características morfológicas do produto final ${ }^{[39-41]}$.

Nos últimos anos, empresas internacionais do setor aeronáutico vêm utilizando essas técnicas, visando compreender e dominar a relação morfologia-processamento-propriedades de compósitos poliméricos utilizados em aeronaves, com o objetivo de redução de custos e a maximização de propriedades. Assim, o estudo reo-cinético de sistemas termorrígidos contribui para a nacionalização do conhecimento estratégico do processamento otimizado de componentes em compósitos poliméricos. O interesse em dominar essa área deve-se ao aumento cada vez maior da utilização de pré-impregnados, com diferentes matrizes termorrígidas em função do uso final do componente, em aeronaves brasileiras, bem como à meta de redução de rejeitos de componentes em compósitos com defeitos introduzidos durante o processamento e, ao contínuo desafio de aumentar a relação empuxo/peso e, com isso, a competitividade das aeronaves nacionais no mercado internacional.

Em função do exposto, e da constante atualização tecnológica exigida no setor aeronáutico, este trabalho tem como objetivo, conhecer os parâmetros cinéticos e reológicos de um sistema de pré-impregnado de resina epóxi (F584), hoje muito usado na indústria aeronáutica. Este estudo foi realizado com o auxílio das técnicas de análises térmicas (DSC e DMA) e reológicas, estabelecendo a ordem de reação e a cinética de cura do sistema em questão e, a partir dos dados obtidos, foi avaliado o ciclo de cura proposto pelo fornecedor do pré-impregnado.

\section{Experimental}

Neste trabalho foi utilizado um tecido de fibra de carbono tipo 8 HS (Harness-Satin) impregnado com o sistema de resina epóxi F584 produzido pela empresa Hexcel Composites, denominado de prepreg. Esse prepreg é utilizado em estruturas primárias de aeronaves e deve ser armazenado em temperaturas inferiores a $18{ }^{\circ} \mathrm{C}$ negativos, podendo permanecer estocado nesta condição de 6 a 12 meses.

\section{Análises por Calorimetria Exploratória Diferencial}

As análises de calorimetria exploratória diferencial foram realizadas em um equipamento Perkin-Elmer Modelo Pyris 1-DSC, previamente calibrado com índio e zinco, sob fluxo constante de nitrogênio $(20 \mathrm{~mL} / \mathrm{min})$ e razão de aquecimento de $2,5{ }^{\circ} \mathrm{C} / \mathrm{min}$. As amostras de prepregs $(5,4$ a $5,7 \mathrm{mg}$ ) foram encapsuladas em um porta-amostra de alumínio do tipo selado. As variáveis relacionadas com a cinética de cura foram obtidas a partir de varreduras dinâmicas de 30 a $300{ }^{\circ} \mathrm{C}$ realizadas sob as razões de aquecimento de 2,$5 ; 5,0$ e $10{ }^{\circ} \mathrm{C} / \mathrm{min}$.

\section{Análises Reológicas}

As análises reológicas foram realizadas em amostras de pré-impregnados de fibras de carbono/epóxi com diâmetro de $25 \mathrm{~mm}$ e espessura de $0,3 \mathrm{~mm}$, utilizando-se um reômetro de tensão controlada da Rheometric Scientific, modelo SR5 , com pratos paralelos. Os ensaios foram realizados à frequiência constante de $0,16 \mathrm{~Hz}$ e tensão de $500 \mathrm{~Pa}$ a diferentes razões de aquecimento: 2,$5 ; 5 ; 10{ }^{\circ} \mathrm{C}$.

\section{Análise Térmica Dinâmico-Mecânica}

Estas análises foram realizadas em um equipamento DMA da TA Instruments, modelo 2980, acoplado a um termo-analisador, em ambiente de ar atmosférico.

As amostras dos prepregs foram cortadas nas dimensões de $40 \mathrm{~mm}$ de comprimento, $13 \mathrm{~mm}$ de largura, e submetidas à varredura dinâmica, simulando etapas de cura que podem ser realizadas em autoclave, ou seja, rampas de aquecimento e isotermas. Assim, as amostras foram aquecidas a $2,5^{\circ} \mathrm{C} / \mathrm{min}$ da temperatura ambiente até 116 ${ }^{\circ} \mathrm{C}$ e mantidas nesta temperatura por $60 \mathrm{~min}$ e, após esta isoterma, o aquecimento foi continuado a $2,5{ }^{\circ} \mathrm{C} / \mathrm{min}$ até $177{ }^{\circ} \mathrm{C}$ e mantido nesta isoterma por $120 \mathrm{~min}$. Foram estabelecidas condições de varredura a $1 \mathrm{~Hz}$ de freqüência e $20 \mu \mathrm{m}$ de amplitude de oscilação sob solicitação mecânica do tipo flexão.

Após a cura dos prepregs, foram realizadas varreduras dinâmicas nas mesmas amostras a $2,5^{\circ} \mathrm{C} / \mathrm{min}$, da temperatura ambiente até $250^{\circ} \mathrm{C}$ para se verificar a extensão da cura e da $T_{g}$ do material curado.

\section{Resultados e Discussão}

\section{Cinética de cura}

Quando um pico é observado em uma curva de DSC, pode-se afirmar que ocorre uma transformação na amostra 
analisada, representada por (1):

$$
B \underset{k}{\longrightarrow} C+\Delta H
$$

onde: $B$ é o material antes da transformação, $C$ é o material após a conversão, $k$ é a constante de velocidade da reação e $\Delta H$ é a entalpia envolvida na reação.

No estudo de cinética química, o alvo de interesse é a taxa de conversão de $B$ em relação ao tempo ou à temperatura. Assim, o grau de conversão $(\alpha)$ pode ser descrito pela Equação (2):

$$
\frac{d \alpha}{d t}=k(1-\alpha)^{n}
$$

onde: $\alpha$ é o grau de conversão da reação química, $n$ é a ordem de reação, $k$ é a constante de velocidade e $d \alpha / d t$ é a derivada primeira de $\alpha$ em relação ao tempo.

A constante de velocidade de reação depende da temperatura de acordo com a relação de Arrhenius (3):

$$
k=A e^{-E_{a} / R T}
$$

onde: $A$ é o fator pré-exponencial, $E_{a}$ é a energia de ativação da reação, $R$ é a constante do gás e $T$ é a temperatura absoluta em kelvin.

Quando as Equações (2) e (3) são combinadas obtem-se a Equação (4):

$$
\frac{d \alpha}{d t}=A \cdot \exp \left(-E_{a} / R T\right)(1-\alpha)^{n}
$$

que apresenta uma variável independente, $t$; duas variáveis dependentes $\alpha$ e $T$, três constantes desconhecidas $A, E_{a}$ e $n$ e a constante do gás, $R$. A Equação (4) possui três graus de liberdade, ou seja, três variáveis. A fim de se obter uma resolução para a equação, durante o processo da reação, uma das variáveis deve ser mantida constante ou estar relacionada com outra variável, assim, as variáveis envolvidas na reação podem ser definidas.

Em uma análise de DSC convencional, a temperatura varia linearmente com o tempo seguindo a relação (5):

$$
T-T(0)=\beta . t
$$

sendo

$$
\beta=\frac{d T}{d t}
$$

onde: $T$ é a temperatura de análise, $T(0)$ é a temperatura inicial, $t$ é o tempo e $\beta$ é a razão de aquecimento.
Além disso, a taxa de reação, $d \alpha / d T$, está diretamente relacionada com a razão de aquecimento de acordo com a Equação (7):

$$
\frac{d \alpha}{d t}=\frac{d \alpha}{d T} \cdot \frac{d T}{d t}=A \cdot \exp \left(-E_{a} / R T\right)(1-\alpha)^{n}
$$

Substituindo a Equação (6) na (7) obtem-se a Equação (8):

$$
\beta \frac{d \alpha}{d T}=A \cdot \exp \left(-E_{a} / R T\right)(1-\alpha)^{n}
$$

Esta equação representa o formato teórico da curva de DSC que é utilizado para o estudo dinâmico da cinética de cura neste trabalho. Os valores de $\alpha$ são obtidos a partir dos valores das entalpias parciais, $\Delta H_{\text {parcial }}$ na tempertura $(\mathrm{T})$, ou seja, como mostra a Equação (9):

$$
\alpha=\frac{\Delta H_{\text {parcial }}}{\Delta H}
$$

Os valores de $\Delta H_{\text {parcial }}$ e $\Delta H$ são obtidos da área sob a curva de DSC.

O método assume que o calor envolvido em um determinado intervalo de tempo é diretamente proporcional ao número de moles reagidos durante o mesmo intervalo de tempo $^{[5,6,18]}$. A taxa de reação é obtida pela divisão do termo dH/dT (variação do fluxo de calor), a uma temperatura $\mathrm{T}$, pela entalpia total $\Delta \mathrm{H}$ (Equação (10)):

$$
\frac{d \alpha}{d T}=\frac{(d H / d T)}{\Delta H}
$$

A Equação (8) agora pode ser reduzida para a forma linear [Equação (11)], aplicando-se o logaritmo natural nos dois lados da equação. A Equação (11) pode ser resolvida a partir de uma regressão multilinear utilizando-se $\ln (\beta d \alpha / d T)$, $-1 / R T$ e $\ln (1-\alpha)$ como variáveis, que podem ser obtidas da curva de DSC. Com isso, os valores de $A, E_{a}$ e $n$ são encontrados.

$$
\ln \left(\beta \frac{d \alpha}{d T}\right)=\ln A-E_{a} / R T+n \cdot \ln (1-\alpha)
$$

Assim, de posse das equações descritas anteriormente e das varreduras dinâmicas do sistema de prepreg de carbono/epóxi realizadas, a diferentes razões de aquecimento, foi possível conhecer os parâmetros envolvidos na cinética de cura, como mostra a Tabela 1 .

Na Tabela 1 as $T_{\text {inicial }}$ e $T_{\text {final }}$ descrevem o intervalo de temperatura em que foi estudada a cinética de cura do prepreg, sempre mantendo o ínicio do estudo em $5 \%$ e o fim em $90 \%$ de conversão, uma vez que é sabido que no ínicio da reação 
Tabela 1. Parâmetros cinéticos obtidos a partir do método dinâmico para três razões de aquecimento: 2,$5 ; 5$ e $10{ }^{\circ} \mathrm{C} / \mathrm{min}$ do sistema de pré-impregnado de fibra de carbono/epóxi (F584).

\begin{tabular}{lcccccc}
\hline Razão de aquecimento $\left({ }^{\mathbf{C}} \mathbf{C} / \mathbf{m i n}\right)$ & $\boldsymbol{T}_{\text {inicial }}\left({ }^{\circ} \mathbf{C}\right)$ & $\boldsymbol{T}_{\text {final }}\left({ }^{\circ} \mathbf{C}\right)$ & $\boldsymbol{\Delta H}(\boldsymbol{J} / \boldsymbol{g})$ & $\boldsymbol{l n} \boldsymbol{A}\left(\mathbf{s}^{-1}\right)$ & $\boldsymbol{E}_{a}(\mathbf{k J} / \mathbf{m o l})$ & $\boldsymbol{n}$ \\
\hline 2,5 & 140 & 242 & -560 & $7,9 \pm 0,7$ & $56,7 \pm 2,7$ & $1,4 \pm 0,1$ \\
5,0 & 145 & 240 & -454 & $9,9 \pm 0,4$ & $62,7 \pm 1,5$ & $1,3 \pm 0,1$ \\
10 & 163 & 244 & -287 & $11,9 \pm 0,5$ & $69,6 \pm 1,9$ & $1,1 \pm 0,1$ \\
\hline
\end{tabular}

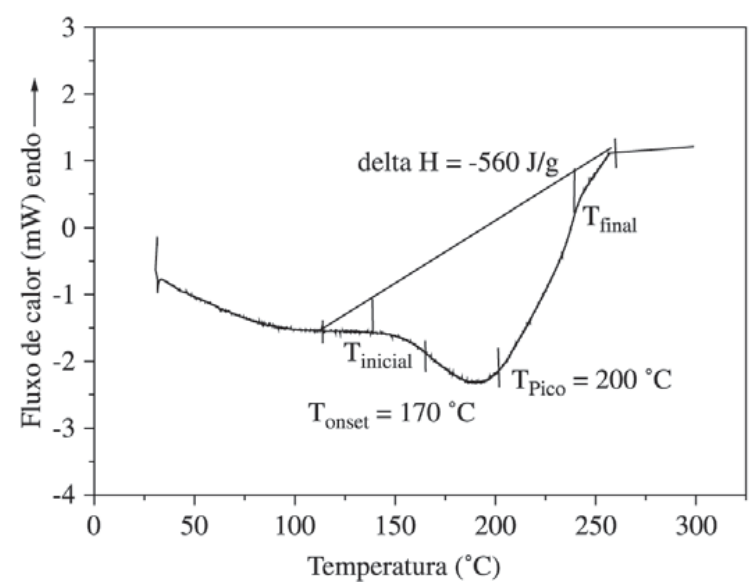

Figura 1. Varredura dinâmica do pré-impregnado de fibra de carbono/epóxi (F584) a $2,5^{\circ} \mathrm{C} / \mathrm{min}$.

o processo é muito rápido e desordenado, sendo difícil estabelecer qualquer parâmetro cinético nesta fase, bem como no final da reação, no qual poucos grupamentos ainda estão por reagir e novamente a descrição dessa fase da cura não é representativa da cinética como um todo.

Com isso, observou-se que o início do evento exotérmico é registrado em temperaturas mais baixas quanto menor for a razão de aquecimento, pois baixas razões de aquecimento permitem a transformação de uma maior parte de grupos químicos relacionados com a reação. Da mesma forma, o calor e a ordem de reação aumentam com a redução da razão de aquecimento. A entalpia e a ordem de reação estão relacionadas com a área da curva obtida no gráfico de DSC, que por sua vez está envolvida diretamente com o desaparecimento de grupos epóxi durante a cura ${ }^{[14]}$.

A ordem de reação de cura fracionada $(\mathrm{n} \sim 1,3)$ encontrada implica em mecanismos complexos de reação. A reação de cura da resina epóxi com endurecedores amínicos leva à formação de amina secundária e de amina terciária. Os grupos hidroxilas gerados durante a reação ou provenientes da adição de solventes ou outros catalisadores, ou pela presença de impurezas e até mesmo de umidade, aceleram significantemente a reação entre o glicidil éter e a amina. No entanto, em todos os casos os grupamentos hidroxila se comportam somente como catalisadores da reação e não como consumidores de grupos epóxi em competição com o grupamento amina ${ }^{[5,11,18]}$. Sendo assim, mecanismos complexos de reação caracterizam a cura da resina epóxi com endurecedores amínicos.

Os valores obtidos para o fator pré-exponencial $\left(\sim 8-12 \mathrm{~s}^{-1}\right)$ e para a energia de ativação $(\sim 57-70 \mathrm{~kJ} / \mathrm{mol})$ estão dentro da faixa prevista na literatura para reações entre os grupamentos epóxi e aminas aromáticas $(50-100 \mathrm{~kJ} / \mathrm{mol})^{[6,7,10-15]}$.

Com o intuito de tentar descrever o que ocorre durante a cura do pré-impregnado na autoclave, durante o processamento de um artefato em compósito, o gráfico de DSC obtido à razão de aquecimento a $2,5^{\circ} \mathrm{C} / \mathrm{min}$ foi a curva escolhida para representar o sistema (Figura 1). Essa razão de aquecimento foi usada como referência por ser a razão utilizada industrialmente na autoclave para a cura desse sistema de pré-impregnado em estudo. Com isso, foi possível obter e avaliar os parâmetros cinéticos utilizados no processamento de compósitos poliméricos a partir de fibras de carbono/epóxi (F584). Isso se faz necessário, uma vez que o conhecimento da cinética de polimerização de prepregs permite avaliar e otimizar os parâmetros de cura desses sistemas, visando melhorar a qualidade do produto final, bem como a redução de custos e rejeitos.

Na Figura 1 pode-se observar um rápido aumento na taxa de reação até aproximadamente a temperatura de pico máximo de conversão $\left(T_{\text {pico }}=200{ }^{\circ} \mathrm{C}\right)$, seguido por um decréscimo exponencial até cessar completamente o evento, que se dá em torno de $250^{\circ} \mathrm{C}$. A área do pico acima da região exotérmica entre a $T_{\text {inicial }}$ e $T_{\text {final }}$ foi utilizada para determinar o grau de conversão, $\alpha$, da resina epóxi em 21 diferentes temperaturas, como mostra a Equação (9). A fim de se verificar a adequabilidade da Equação (11) na descrição dos dados cinéticos obtidos foi construído o gráfico de Arrhenius da Figura 2, onde se pode observar que existe concordância entre os pontos experimentais (círculos) e o modelo téorico (reta). Esse resultado mostra que o sistema de pré-impregnado estudado pode ser descrito pela cinética de ordem $n$ e ser avaliado tanto pelo método dinâmico como pelo isotérmico ${ }^{[5]}$.

O ciclo de cura empregado industrialmente na autoclave compõe-se de 4 etapas, sendo: aquecimento de 30 a $116^{\circ} \mathrm{C}$ a $2,5{ }^{\circ} \mathrm{C} / \mathrm{min}$; seguido de uma isoterma a $116{ }^{\circ} \mathrm{C}$ por 60 min; novo aquecimento de 116 a $177{ }^{\circ} \mathrm{C}$ também a $2,5^{\circ} \mathrm{C} /$ min e por último uma isoterma a $177^{\circ} \mathrm{C}$ por no mínimo 120 min. A partir desses dados fez-se uma simulação matemática do grau de conversão da resina epóxi obtida em cada etapa.

O gráfico da Figura 3 apresenta o grau de conversão do pré-impregnado de carbono/epóxi em função da temperatura no intervalo de tempo de 34,4 min, que é o tempo necessário para se passar de 30 a $116^{\circ} \mathrm{C}$ à razão de aquecimento de $2,5^{\circ} \mathrm{C} / \mathrm{min}$. Esse gráfico foi obtido baseando-se na Equação (12), fixando-se o tempo e escolhendo-se os dois limites de temperatura: 


$$
\alpha=1-\left[1-(1-n) \cdot \text { A.t. } \exp \left(-E_{a} / R T\right)\right]^{(1 /(1-n))}
$$

Com o gráfico da Figura 3 pode-se observar que na primeira etapa da autoclave o pré-impregnado atinge um grau de conversão de, aproximadamente, $16 \%$. Nesta etapa inicia-se a abertura dos anéis epóxi gerando reações entre grupos epóxi e uma amina primária, que por sua vez levam à formação de aminas secundárias e terciárias, bem como à formação de grupos hidroxilas que aceleram a reação de polimerização $o^{[5]}$. Nesta etapa tem início, também, o processo de gelificação com a formação incipiente de uma rede de massa molar infinita, onde a viscosidade à taxa de cisalhamento tendendo a zero também torna-se infinita, dando origem ao comportamento viscoelástico do sistema. O sistema passa de um líquido viscoso a um gel elástico ${ }^{[23-26]}$.

A segunda etapa da autoclave é mostrada pelo gráfico da Figura 4 que descreve o grau de conversão em função do tempo para uma temperatura isotérmica escolhida, conforme apresentado pela Equação (12) anterior, só que agora o gráfico é construído com base na temperatura isotérmica e

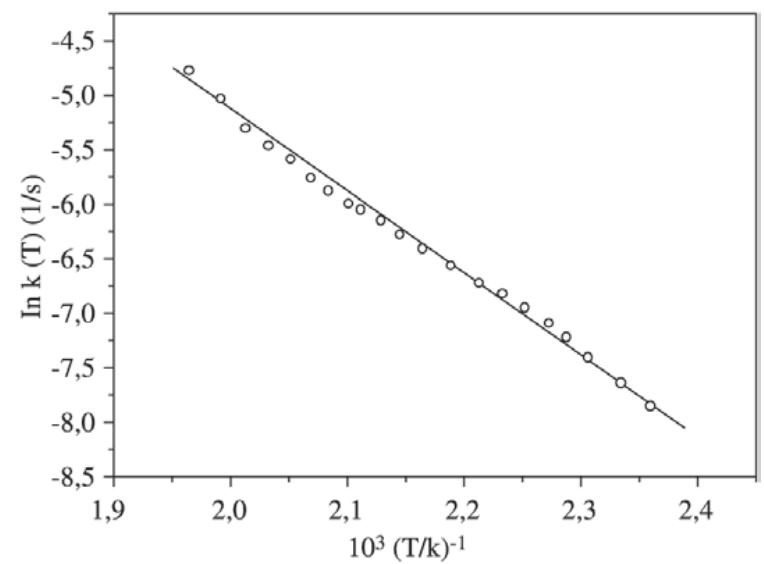

Figura 2. Gráfico de Arrhenius do prepreg de carbono/epóxi, F584, para os dados obtidos a partir da razão de aquecimento de $2,5^{\circ} \mathrm{C} / \mathrm{min}$.

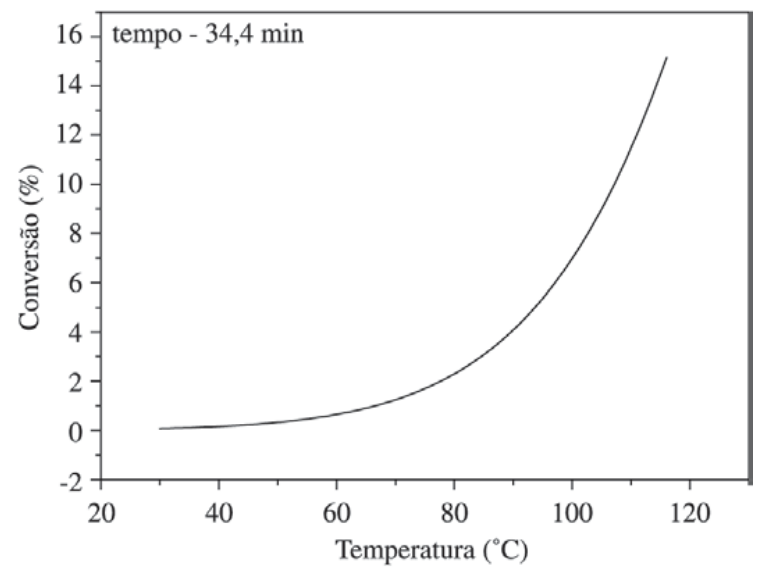

Figura 3. Grau de conversão do prepreg de carbono/epóxi, F584, em função da temperatura na faixa de 30 a $116^{\circ} \mathrm{C}$. de dois limites de tempo definidos.

$\mathrm{Na}$ Figura 4 verifica-se que o processo de cura atinge $25 \%$ de conversão. Nesta fase, provavelmente, existe a presença de algumas moléculas que estão atingindo a gelificação e outras que já passaram pelo processo. Assim, o processo de cura continua mais lentamente, porque a mobilidade do sistema fica mais restrita, devido ao aumento da densidade de ligações cruzadas.

A Figura 5 mostra a conversão de, aproximadamente, $75 \%$ que ocorre na faixa de temperatura de 116 a $177^{\circ} \mathrm{C}$ no tempo de $24,4 \mathrm{~min}$. Esta etapa sugere que o processo de vitrificação está presente, ou seja, a fase na qual a reação química é resfriada durante o ciclo de cura, sendo que a resina ainda não atingiu o estado de cura total.

E finalmente na Figura 6, pode-se verificar que o sistema atinge próximo de $100 \%$ de cura a partir de aproximadamente $80 \mathrm{~min}$. Entretanto, se faz necessário deixar as peças de diversas espessuras manufaturadas com o pré-impregnado de fibra de carbono/epóxi no mínimo por $120 \mathrm{~min}$ a $177^{\circ} \mathrm{C}$, para garantir que toda a estrutura se encontre completamente curada e atinja, dessa forma, o melhor desempenho das

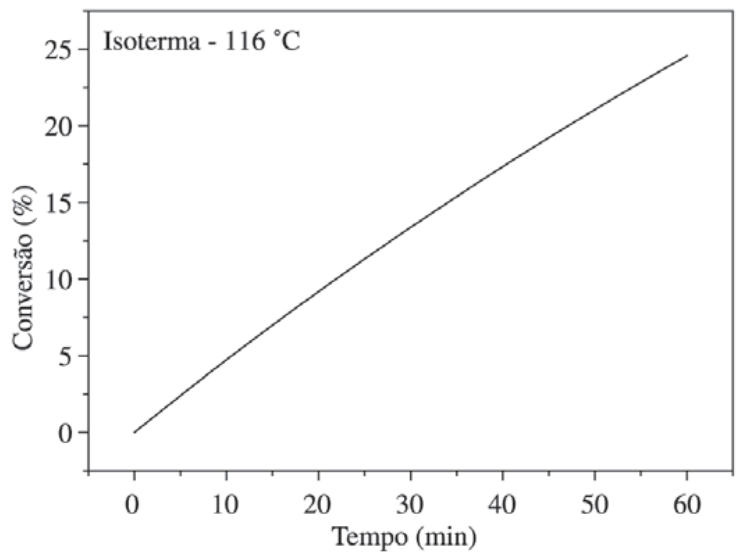

Figura 4. Grau de conversão do prepreg de carbono/epóxi, F584, em função do tempo para isoterma a $116^{\circ} \mathrm{C}$.

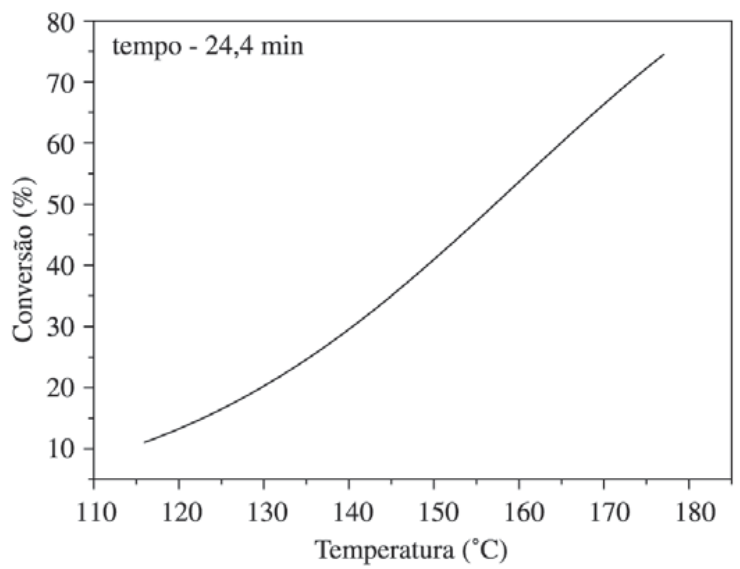

Figura 5. Grau de conversão do prepreg de carbono/epóxi, F584, em função da temperatura na faixa de 116 a $177^{\circ} \mathrm{C}$. 


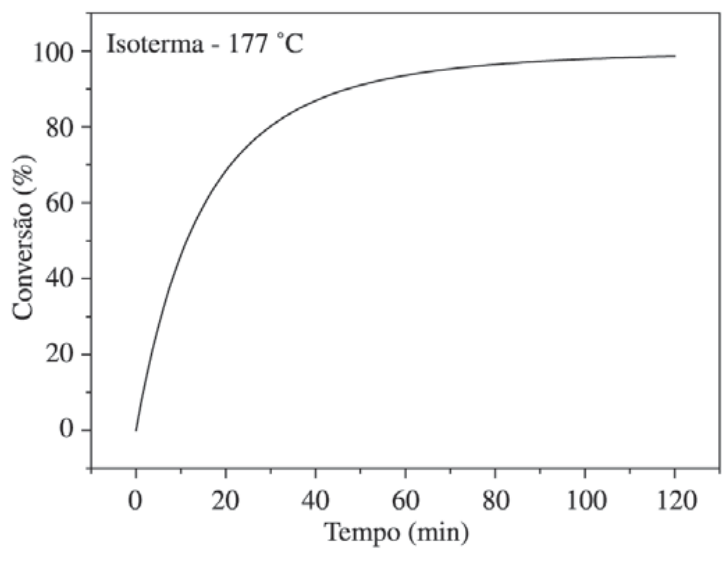

Figura 6. Grau de conversão do prepreg de carbono/epóxi, F584, em função da tempo para a isoterma a $177^{\circ} \mathrm{C}$.

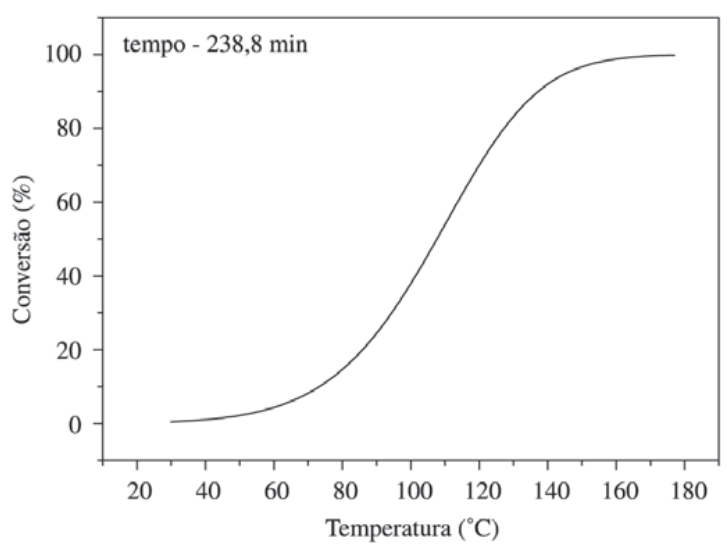

Figura 7. Grau de conversão total do prepreg de carbono/epóxi, F584, em função da temperatura de cura na autoclave.

propriedades mecânicas do compósito polimérico.

A Figura 7 resume todas as etapas descritas anteriormente, mostrando a evolução da reação de cura em todo o ciclo de cura empregado na autoclave no processamento dos compósitos poliméricos.

\section{Análise Reológica}

A Figura 8 apresenta as curvas viscosimétricas do préimpregnado de fibra de carbono/epóxi após ser submetido às análises reológicas. Como pode ser observado, a evolução da temperatura reflete inicialmente na diminuição da viscosidade complexa $\left(\eta^{*}\right)$ até ser atingida uma temperatura a partir da qual há o início do processo de cura do pré-impregnado com a formação de uma rede tridimensional. Esta diminuição de viscosidade ocorre devido à destruição gradativa das forças de interação (van der Waals) existentes na resina ainda não curada, sendo que esta estrutura pode ainda ser destruída com o aumento da taxa de cisalhamento e facilmente recomposta quando esta velocidade diminui.

A partir da análise da Figura 8 e da Tabela 2, foi constatado que a região de viscosidade mínima ocorreu em tempe-

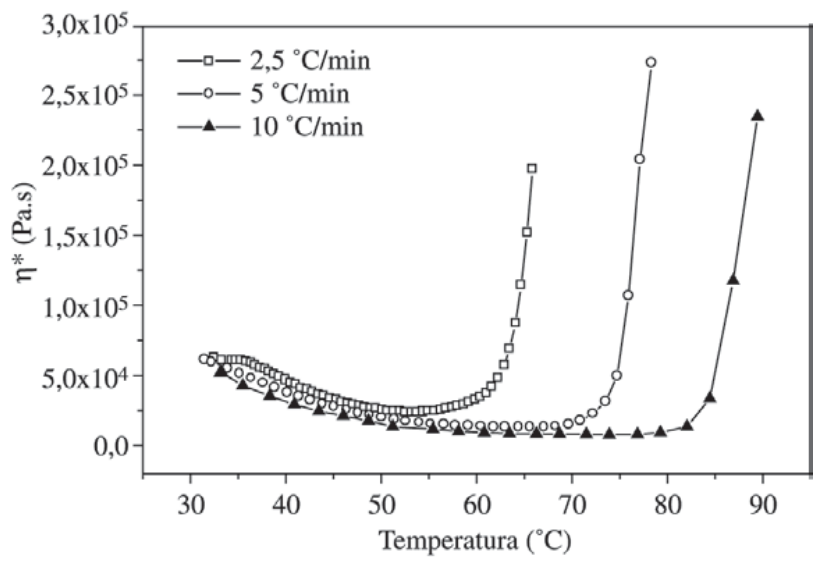

Figura 8. Evolução da viscosidade complexa do prepreg de carbono/epóxi, F584, com a temperatura a diferentes razões de aquecimento.

raturas mais elevadas com o aumento da razão de aquecimento devido, provavelmente, à uma cinética de cura mais lenta ocasionada por um aquecimento mais rápido da amostra analisada. Entretanto, o valor da viscosidade mínima diminuiu com o aumento da razão de aquecimento, devido às temperaturas mais elevadas favorecerem o enfraquecimento das interações secundárias existentes na estrutura da matriz polimérica.

Durante a análise viscosimétrica verificou-se uma evolução significativa da viscosidade complexa após a passagem pela região de mínimo de viscosidade, caracterizando um aumento contínuo da componente elástica, devido à uma rede formada por partículas ou agregados interconectados por interações elásticas, caracterizando a passagem de um sistema Newtoniano a um sistema viscoelástico. Durante a cura, à medida que a reação ocorre, as cadeias do polímero crescem, depois se ramificam e em função da densidade de ligações cruzadas, sua flexibilidade e mobilidade diminuem.

A Figura 8 e a Tabela 2 evidenciam também a influência da razão de aquecimento utilizada no começo da evolução da viscosidade complexa para a amostra analisada. Como pode ser observado, a evolução da viscosidade complexa começou a ocorrer a 60,70 e $80^{\circ} \mathrm{C}$ para as razões de aquecimento de 2,$5 ; 5$ e $10{ }^{\circ} \mathrm{C} / \mathrm{min}$, respectivamente. Esta diferença significativa entre as temperaturas de início de evolução das curvas de viscosidade complexa com o aumento da razão de aquecimento, era esperado devido a um tempo maior de residência favorecer o início da cinética de cura do sistema analisado, ocasionando uma cura mais homogênea e controlada quando comparado com elevadas razões de aquecimento.

O aumento gradativo da viscosidade observado no início da análise deve-se a uma lenta reestruturação do sistema. Entretanto, com a simples observação da evolução da viscosidade complexa não é possível determinar o tempo de gel com precisão. No acompanhamento viscoelástico, o tempo de gel pode ainda ser obtido por dois outros métodos: pelo cruzamento entre $G$ ' (módulo de armazenamento) com $G$ " (módulo de perda) ou pela variação da $\tan \delta$ (amortecimen- 
Tabela 2. Variação das propriedades reológicas do prepreg de carbono/epóxi, F584, com o aumento da razão de aquecimento.

\begin{tabular}{lccc}
\hline Variáveis reológicas & $\mathbf{2 , 5}{ }^{\circ} \mathbf{C} / \mathbf{m i n}$ & $\mathbf{5 , 0}{ }^{\circ} \mathbf{C} / \mathbf{m i n}$ & $\mathbf{1 0}{ }^{\circ} \mathbf{C} / \mathbf{m i n}$ \\
\hline$\eta^{*}$ inicial (Pa.s) & 65.000 & 61.000 & 63.000 \\
$\eta^{*}$ mínima (Pa.s) & 25.400 & 15.690 & 10.790 \\
Temp. de $\eta^{*}$ mínima $\left({ }^{\circ} \mathrm{C}\right)$ & 55 & 62 & 72 \\
$T_{\text {gel }}$ pelo método da $\eta^{*}\left({ }^{\circ} \mathrm{C}\right)$ & 62 & 72 & 84 \\
$T_{\text {gel }}$ pelo método de $\left.G^{\prime} \mathrm{x} G{ }^{\prime}{ }^{\circ}{ }^{\circ} \mathrm{C}\right)$ & 65 & 77 & 87 \\
$T_{\text {gel }}$ pelo método de $\tan \delta\left({ }^{\circ} \mathrm{C}\right)$ & 65 & 76 & 87 \\
Valor máximo de $\tan \delta$ & 2,3 & 3,2 & 4,2 \\
Temperatura de $\tan \delta\left({ }^{\circ} \mathrm{C}\right)$ & 60 & 71 & 82 \\
\hline
\end{tabular}

to), na qual a temperatura de cura começa a ocorrer para valores de $\tan \delta$ igual a 1 ( $\left.\tan \delta=G^{\prime \prime} / G^{\prime}\right)$.

Devido aos compósitos poliméricos serem materiais viscoelásticos (ou seja, possuem uma resposta composta de uma parte elástica e outra viscosa) e assumindo que esses materiais foram submetidos a uma deformação oscilatória de cisalhamento de pequena amplitude $(1 \mathrm{rad} / \mathrm{s})$, as tensões resultantes oscilarão com a mesma freqüência $\omega$, mas não estarão em fase com a deformação, exibindo, então, um comportamento viscoelástico linear. Assim, $G$ ' está associado à componente em fase com a deformação, ou seja, está associado à contribuição elástica ou armazenamento de energia em cada ciclo e $G$ ” está associado à componente fora de fase $^{[39-41]}$.

Desta forma, a Figura 9 e a Tabela 2 apresentam a evolução de $G$ ' e $G$ ” com a temperatura das amostras de prepreg de fibra de carbono/epóxi analisadas a diferentes razões de aquecimento $\left(2,5 ; 5\right.$ e $\left.10{ }^{\circ} \mathrm{C} / \mathrm{min}\right)$. Todas as curvas foram obtidas à frequiência de $0,16 \mathrm{~Hz}$ e desta forma $G^{\prime}$ permanece inferior a $G$ ”, confirmando que as características de um sólido elástico não são atingidas antes da região de gel. A partir deste gráfico foi novamente observado que os valores encontrados para a temperatura de gelificação aumentaram com o aumento da razão de aquecimento, confirmando os resultados obtidos via análise viscosimétrica. Entretanto, os valores de $T_{g e l}$ obtidos via cruzamento de $G$ ' $\operatorname{com} G$ ” foram ligeiramente superiores aos encontrados via análise viscosimétrica ( $\sim 5 \%$ maiores).

A Figura 10 e a Tabela 2 apresentam a variação de $\tan \delta$ com a temperatura das amostras de fibra de carbono/epóxi analisadas a diferentes razões de aquecimento $(2,5 ; 5 \mathrm{e}$ $10{ }^{\circ} \mathrm{C} / \mathrm{min}$ ). A partir destas curvas foi observado que tanto os valores máximos de $\tan \delta$ quanto a temperatura em que estes valores atingiram seus valores máximos aumentaram com o aumento da razão de aquecimento. Este fato ocorreu devido às elevadas temperaturas favorecerem o escoamento viscoso do prepreg, ou seja, valores mais elevados de $G$ ”.

Com base nas informações dinâmicas obtidas via análises reológicas, pode-se concluir que a razão de aquecimento mais adequada para a obtenção de um compósito estrutural foi a de $2,5^{\circ} \mathrm{C} / \mathrm{min}$, devido à esta razão de aquecimento proporcionar uma cura a uma temperatura mais baixa quando comparada a 5 e $10{ }^{\circ} \mathrm{C} / \mathrm{min}$, proporcionando uma cura mais homogênea e controlada.

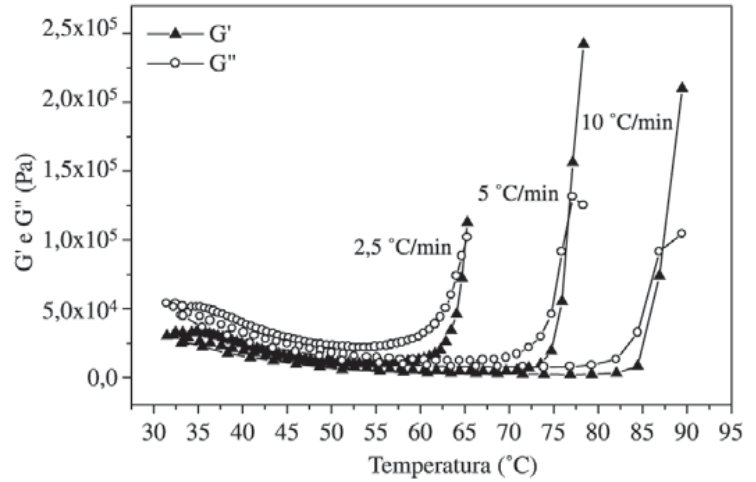

Figura 9. Evolução dos módulos viscoso e elástico do prepreg de carbono/epóxi, F584, com a temperatura a diferentes razões de aquecimento.

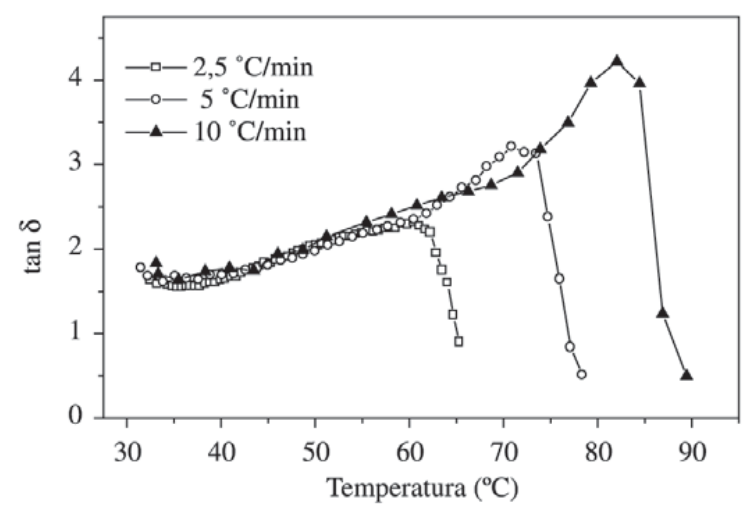

Figura 10. Variação da tan $\delta$ do prepreg de carbono/epóxi, F584, com a temperatura a diferentes razões de aquecimento.

\section{Análise Térmica Dinâmico-Mecânica (DMTA ou DMA)}

Na Figura 11 está apresentada a curva de DMA da amostra de prepreg F584 submetida a rampas de aquecimento e isotermas a $116{ }^{\circ} \mathrm{C}$ e $177{ }^{\circ} \mathrm{C}$. De acordo com as curvas de DMA, durante a varredura ocorre um aumento significativo no módulo de armazenamento $E$ ' do material, indicando que o processo de cura ocasiona o aumento da rigidez da amostra, como decorrência do aumento da densidade de ligações cruzadas da matriz epóxi, ocasionando o enrijecimento da matriz de resina presente no prepreg.

Na Figura 4 referente ao grau de conversão do prepreg F584 foi observado que após a isoterma a $116^{\circ} \mathrm{C}$, a porcen- 
tagem de cura do material é cerca de $25 \%$, e quando comparada com a curva de DMA (Figura 11) é observado que esta porcentagem de cura, como já esperado, ainda não ocasiona aumento significativo na rigidez do material (módulo E'). No entanto, é importante ressaltar que a isoterma a $116^{\circ} \mathrm{C}$ é de primordial importância na cura do material, já que nesta temperatura ocorre uma etapa de entrecruzamento da matriz epóxi, proporcionando condições adequadas para a aplicação da pressão e vácuo durante a moldagem na autoclave minimizando, desta maneira, a formação de defeitos tipo vazios e regiões pobres em resina no compósito curado, que podem ser ocasionados pela liberação de voláteis ou perda de resina durante o processo de cura.

Após o término desta primeira etapa de cura, ou seja, após a conclusão da isoterma a $116^{\circ} \mathrm{C}$, é observado o grande aumento no $E^{\prime}$ com o prosseguimento da varredura até se atingir a segunda isoterma a $177^{\circ} \mathrm{C}$, revelando que na matriz de resina ocorreram reações de entrecruzamento, que elevaram o E' para um valor máximo (aproximadamente $6000 \mathrm{MPa}$ ). Este comportamento de aumento do $E^{\prime}$ durante o entrecruzamento também foi relatado na literatura ${ }^{[42,43]}$. Estas observações estão de acordo com a Figura 6 referente ao grau de conversão do prepreg, que também mostra que a isoterma a $177{ }^{\circ} \mathrm{C}$, favorece o entrecruzamento da matriz epóxi, chegando a próximo de $100 \%$ de conversão.

Ainda, na Figura 11 é observado que durante a isoterma a $177^{\circ} \mathrm{C}$, o prepreg mostra um pico de transição vítrea $\left(T_{g}\right)$. Apesar da Figura 11 ser relativa à cura do prepreg, pode-se observar um pico de transição vítrea, o qual é revelado pelo pico no módulo de perda ( $E$ ") e na curva tan delta. Comparando-se este dado com o resultado obtido nas análises por DSC, que mostram que o prepreg já encontra-se curado após 80 min de patamar a $177^{\circ} \mathrm{C}$, o pico de transição deve-se ao material já curado. Este comportamento está relacionado à movimentação de partes mais livres da cadeia.

Na Figura 12 está apresentada a curva de DMA da amostra de prepreg curada previamente no DMA e que, em seguida, foi submetida à varredura dinâmica no mesmo equipamento para se confirmar a cura do material pelo perfil da curva obtida e comparar com a Figura 7 do DSC, a qual

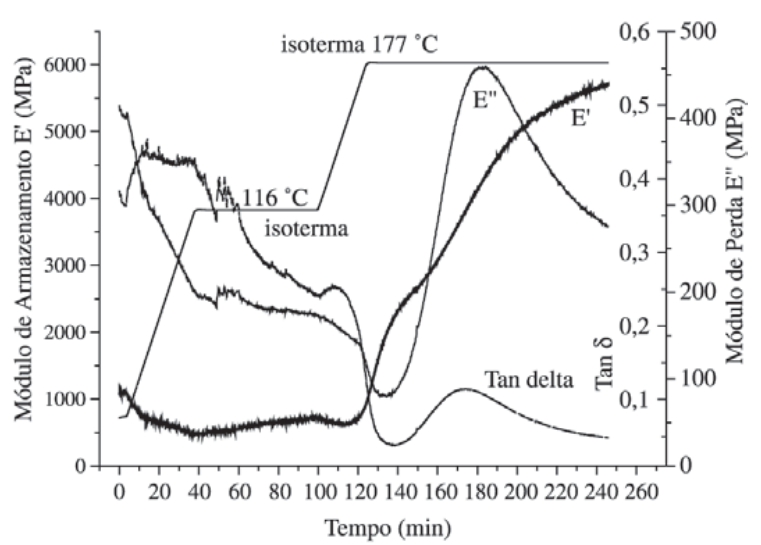

Figura 11. Curva de DMA do prepreg F584, varredura de cura a $2,5^{\circ} \mathrm{C} / \mathrm{min}$. revelou que a matriz epóxi do prepreg já estava totalmente curada.

De acordo com a Figura 12 é observado que as etapas de cura realizadas anteriormente na amostra de prepreg foram realmente suficientes para ocasionar a cura da matriz, já que o módulo de armazenamento $E$ ', não mais apresenta característica de enrijecimento do material à medida que a temperatura aumenta. Outra característica relacionada ao entrecruzamento da matriz está relacionada ao perfil agudo do pico da curva $E$ ”, não apresentando cura adicional ou residual, a qual poderia ocasionar um alargamento deste pico $^{[42,44]}$.

Baseando-se na Figura 12 pode-se considerar que o material apresenta pequeno decréscimo no módulo $E^{\prime}$ até a temperatura de cerca de $150{ }^{\circ} \mathrm{C}$, praticamente não ocasionando mudanças em suas propriedades mecânicas. A partir de $150^{\circ} \mathrm{C}$, ocorre uma queda mais acentuada na curva de $E$ ' e o aumento nas curvas de $E$ " e tan delta, caracterizando a região da temperatura de transição vítrea $\left(T_{g}\right)$. Considerando-se, neste trabalho, a determinação da temperatura de transição vítrea pelo máximo do módulo de perda ( $E$ ”), pode-se verificar que a $T_{g}$ da resina F584 (contida no prepreg curado no DMA) é igual a $201{ }^{\circ} \mathrm{C}$.

Na Figura 13 está apresentada a curva de DMA do compósito moldado a partir de várias camadas de prepreg F584 reforçado com tecido de fibras de carbono $8 \mathrm{HS}$, ou seja, exatamente o material do prepreg analisado neste trabalho. Este compósito foi obtido em autoclave, submetido às mesmas etapas de cura realizadas por análises de DSC e DMA e descritas anteriormente.

Na curva de DMA do compósito (Figura 13) pode-se observar que a temperatura de transição vítrea da matriz epóxi F584 curada é de $177^{\circ} \mathrm{C}$ (considerando-se $E$ " para a determinação da $T_{g}$ ), estando próxima à $T_{g}$ informada pelo fabricante do prepreg, que é de $\left.171^{\circ} \mathrm{C}^{[45]}\right]^{g}$.

Assim, comparando-se as curvas de DMA do prepreg (Figura 12) e do compósito (Figura 13) é observado que a $T$ de uma camada de prepreg curada é superior à $T_{g}$ do compósito obtido após o empilhamento de várias camadas, pois neste caso durante a varredura no DMA a matriz curada

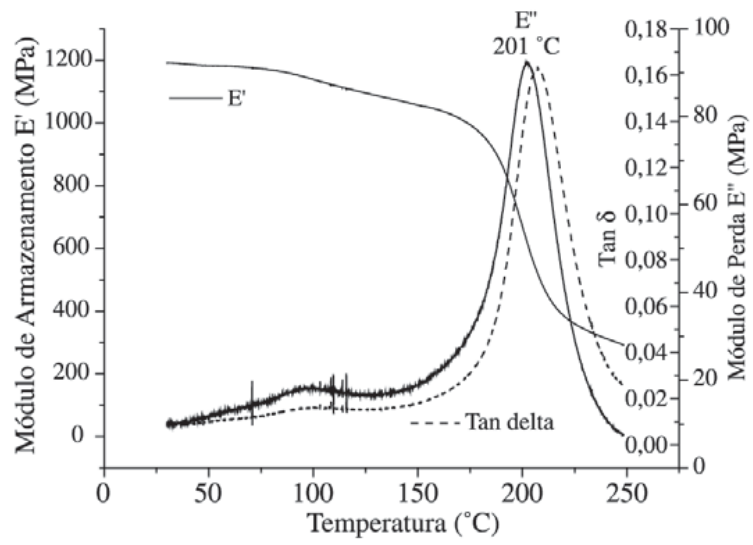

Figura 12. Curva de DMA do prepreg F584 previamente curado; varredura dinâmica a $2,5^{\circ} \mathrm{C} / \mathrm{min}$. 


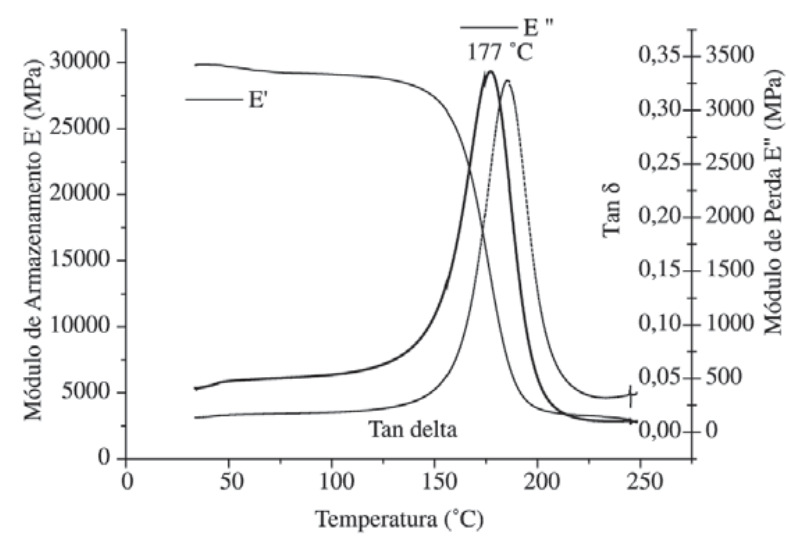

Figura 13. Curva de DMA do compósito F584/tecido de fibra de carbono $8 \mathrm{HS}$; varredura dinâmica a $2,5^{\circ} \mathrm{C} / \mathrm{min}$.

do compósito apresenta diversas interações e movimentações intra e intermoleculares na região de transição vítrea, as quais também são influenciadas pelas várias camadas existentes no compósito e na difusão de calor durante a cura, ocasionando um abaixamento da $T_{g}{ }^{[46,47]}$.

Ainda, analisando-se a Figura 13, observa-se que o módulo de armazenamento $E$ ' praticamente não apresenta decréscimo até atingir a temperatura de cerca de $125^{\circ} \mathrm{C}$, quando a partir desta começa a apresentar contínuo decréscimo, alterando as propriedades mecânicas do compósito. Pode-se verificar que a partir de $125{ }^{\circ} \mathrm{C}$ o compósito entra na região de transição vítrea, ocasionando aumento no módulo de perda $E$ " e na curva de tan delta. Assim, pela análise da curva de DMA pode-se, considerar $125^{\circ} \mathrm{C}$ como temperatura máxima de aplicação intermitente para este material, sem alteração de suas propriedades.

\section{Conclusões}

O uso da técnica de calorimetria exploratória diferencial mostrou-se adequada ao estudo da cinética de cura de sistemas de pré-impregnado de fibra de carbono/resina epóxi (F584). A formulação estudada apresentou cinética de cura de ordem $n$ em torno de 1,3. O valor obtido para a energia de ativação ( 57-70 kJ/mol) está dentro da faixa descrita pela literatura.

As análises dinâmicas a partir de ensaios reológicos e por DSC foram concordantes. A partir das curvas reológicas analisadas foi constatado que as regiões de viscosidade mínima e viscosidade complexa, temperatura de gelificação e valor de tan $\delta$ do pré-impregnado estudado, ocorreram em temperaturas mais elevadas com o aumento da razão de aquecimento, devido às temperaturas mais elevadas resultarem em uma cinética de cura mais rápida. Com base nestas informações, pode-se concluir que a razão de aquecimento mais adequada para a obtenção de um compósito estrutural, a partir do sistema estudado e das análises realizadas neste trabalho, é de $2,5^{\circ} \mathrm{C} / \mathrm{min}$, devido à esta razão de aquecimento proporciar processo de cura a uma temperatura mais baixa, quando comparada a 5 e $10{ }^{\circ} \mathrm{C} / \mathrm{min}$, favorecendo uma cura mais homogênea e controlada.

Assim, a partir dos estudos dinâmicos realizados no presente trabalho (DSC, reologia e DMA), pode-se concluir que, o ciclo de cura, hoje, utilizado na manufatura dos compósitos poliméricos de fibra de carbono/resina epóxi (F584) mostrou-se adequado para obtenção de peças aeronáuticas com alto grau de cura, atingindo, conseqüentemente, as propriedades mecânicas desejadas.

\section{Agradecimentos}

Os autores agradecem à FAPESP pelo suporte financeiro (02/01808-7; 02/01288-3; 02/02057-5); ao CNPq (300599/96); às empresas Hexcel Composites e EMBRAER por terem cedido os pré-impregnados utilizados no presente trabalho, bem como à dpUNION pelo empréstimo do equipamento de DMA modelo 2980 da TA Instruments.

\section{Referências Bibliográficas}

1. Schwartz, M.M. Composite Materials Properties, Nondestructive Testing and Repair, vol. I, Prentice Hall Inc, New Jersey, USA, 1997.

2. Pardini, L.C.; Peres, R.J.C. Polímeros: Ciência e Tecnologia, abr/jun, p. 32-42, 1996.

3. ASM International Comitee. Engineered Materials Handbook, in: Engineering Plastics USA:ASM International, v. 2, 1987.

4. Costa, M.L. Estabelecimento de parâmetros de processamento de compósitos estruturais via análises térmica e viscosimétrica, Dissertação de Mestrado, ITA, São José dos Campos, p.142, Brasil, 1998.

5. Costa, M.L.; Pardini, L.C.; Rezende, M.C. Polímeros: $\mathrm{Ci}$ ência e Tecnologia. Abr/Jun, 2, p. 37-44, 1999.

6. Costa, M.L.; Pardini, L.C.; Rezende, M.C. Química Nova, Maio/Junho, 23, 3, p. 320-325, 2000.

7. Costa, M.L.; Pardini, L.C.; Rezende, M.C. Journal of Thermal Analysis, submetido.

8. Ghaemy, M.; Riahy, M.H. European Polymer Journal, 32, 10, p. 1207-1212, 1996.

9. Morgan, R.J.; Shin, E.E.; Rosenberg, B.; Jurek, A. Polymer 38, 3, p. 639-646, 1997.

10. Shim, S.B.; Seferis, J.C.; Eom, Y.S.; Shim, Y.T. Thermochimica Acta, 291, p. 73-79, 1997.

11. Barral, L.; Cano, J.; López, J.I.; López-Bueno, P.N.; Torres, A.; Ramirez, C.; Abad, M.J. Thermochimica Acta 344, p.127-136, 2000.

12. Wingard, C.D. Thermochimica Acta, 357, p. 293-301, 2000.

13. Blest, D.C.; Duffy, B.R.; McKee, S.; Zulkifle, A.K. Composites Part A: Applied Science and Manufacturing 30, p. 1289-1309, 1999.

14. Varley, R.J.; Hodgkin, J.H.; Hawthorne, D.G.; Simon, G.P.; McCulloch, D. Polymer, 41, p. 3425-3436, 2000.

15. Shin, D.D.; Hahn, H.T. Composites Part A: Applied Science and Manufacturing 31, p.991-999, 2000.

16. Hayes, B.S.; Gilbert, E.N.; Seferis, J.C. Composites Part A: Applied Science and Manufacturing, 31, p. 717- 
725, 2000.

17. Lange, J.; Altmann, N.; Kelly, C.T.; Halley P.J. Polymer, 41, p. 5949-5955, 2000.

18. Prime, R.B. Thermosets, in the thermal characterization of polymeric materials, London, Academic Press, p. 435-569, 1981.

19. Woo, E.M.; Mao, K.L. Composites part A 27A, p. 625631, 1996.

20. Buggy, M.; Temimhan, T.; Braddell Journal of Materials Processing Technology, 56, p. 292-301, 1996.

21. White, S.R.; Kim, Y.K. Composites part A, 27A, p. 219227, 1996.

22. Lee, J.Y.; Choi, H.K.; Shim, M.J.; Kim, S.W. Thermochimica Acta 343, p. 111-117, 2000.

23. Botelho, E.C.; Scherbakoff, N.; Rezende, M.C. Journal of Avanced Materials, 33, 4, p. 44-51, 2001.

24. Botelho, E.C.; Scherbakoff, N.; Rezende, M.C. Polímeros: Ciência e Tecnologia, 1, p. 59, 1999.

25. Botelho, E.C.; Rezende, M.C. Polímeros: Ciência e Tecnologia 10, 2, p. E4, 2000.

26. Botelho, E.C. Estudo reológico de resinas fenólicas e furfurílica na obtenção de materiais carbonosos, Dissertação de mestrado, Instituto Tecnológico de Aeronáutica (ITA), São José dos Campos-SP, 1998.

35. Mimura, K; Ito, H.; Fujioka, H. Polymer, 42, p. $9223-$ 9233, 2001.

36. Saalbrink, A.; Lorteije, A.; Peijs, T. Composites Part A, 29A, p. 1243-1250, 1998.

37. Bretas, R.E.S.; D’Avila, M.A. Reologia de Polímeros
Fundidos, Editora da UFSCar, FAPESP, São Carlos, 2000.

38. Sichina, W. J. Applications of the Time-Temperature Superposition Princile. Catálogo da Du Pont. Applications Brief, 1999.

39. Tajima, Y.A. Polymer Composites, 3, 3, p.162-169, 1988.

40. Boll, D.J.; Mottee, B.; Bascom, W.D. Journal of Composites Technology \& Research, 8, 2, p. 48-53, 1986.

41. Laza, J.M.; Julian C.A.; Larrauri, E.; Rodriguez, M.; Leon, L.M. Polymer, 40, p. 35-45, 1998.

42. Shim, S.-B.; Seferis, J.C.; Eom, Y.S.; Shim, Y.T. Thermochimica Acta, 291, p. 73-79, 1997.

43. Wingard, C.D. Thermochimica Acta, 357-358, p. 293 $301,2000$.

44. Hayes, B.S.; Gilbert, E.N.; Seferis, J.C. Composites: Part A 31, p.717-725, 2000.

45. Internet: http://www.hexcelcomposites.com/products/ prepregs/index.html.

46. Murayama, T. Dynamic Mechanical Analysis of Polymeric Material, 2.ed. Amsterdam, Elsevier Scientific Publishing Company, 1982.

47. Hatakeyama, T.; Quinn, F.X. Thermal Analysis Fundamentals and Applications to Polymer Science, England, John Wiley \& Sons, 1994.

Recebido: 06/02/2003

Aprovado: 20/05/2003 